\title{
Effect of Preemptive Intravenous Ibuprofen on propofol Injection Pain
}

\author{
Nilay Taş ${ }^{1 *}$, Ali Altınbaş ${ }^{2}$, Ebru Çanakçı1 ${ }^{1}$ and Zübeyir Cebeci ${ }^{1}$ \\ ${ }^{1}$ Department of Anesthesiology and Reanimation, Ordu University, Turkey \\ ${ }^{2}$ Department of Anesthesiology and Reanimation, Ordu University Training and Research Hospital, Turkey
}

Submission: March 30, 2018; Published: April 13, 2018

*Corresponding author: Nilay Taş, Department of Anesthesiology and Reanimation, Ordu University School of Medicine, Ordu, Turkey, Email: drnilanest@hotmail.com

\section{Abstract}

Aims: The aim of this study is to show whether intravenous ibuprofen administered is effective in preventing propofol injection pain.

Methods and Material: The study included 120 adult patients. Patients in the ibuprofen group were administered $400 \mathrm{mg}$ ibuprofen as intravenous infusion. Patients in the control group were administered physiologic serum, while patients in the lidocaine group were administered $2 \%$ lidocaine intravenously. After 30 seconds the tourniquet was released and $1 / 4$ of the propofol dosage necessary for induction was administered. Then pain in the arm was questioned and arm pull reactions were assessed. Statistical analysis used: The Chi-square test or Fisher's exact test, Student - $t$ test and the one way ANOVA test were used.

Results: In Group C there was no patient with pain score of 0, while this rate was 70\% in Group L and 20\% in Group I ( $\mathrm{p}<0.05$ ). In $52.5 \%$ of patients in Group I there was mild pain observed, and this result was found to be significantly high compared to Group C and Group L ( $\mathrm{p}<0.001$ ). In Group C the number of patients feeling moderate and severe levels of pain was significantly high compared with Group L and Group I ( $\mathrm{p}<0.001$, $\mathrm{p}<0.05)$. In terms of arm pull reactions $85 \%$ of patients in Group $\mathrm{L}$ and $70 \%$ of patients in Group I had no reaction observed, and this result was significantly high compared to Group C $(17.5 \%)(\mathrm{p}<0.001)$.

Conclusions: 400 mg ibuprofen administered had positive effect on reducing propofol injection pain.

Keywords: Propofol; Injection pain; Ibuprofen; Preemptive analgesia

\section{Introduction}

Propofol (2,6 di-isopropyl phenol) is an anesthetic agent commonly used in current anesthesia administration due to many advantages like short duration of effect, ensuring smooth induction, rapid recovery and easy titration [1]. In spite of many superior qualities, injection pain during intravenous (i.v.) administration causes serious rates of discomfort among patients. According to research, the risk of incidence for propofol injection pain varies between $28-90 \%$ for adults [2]. Though injection pain is not a life-threatening complication of anesthesia, it remains an unpleasant situation associated with anesthesia in the minds of patients [3]. Methods such as the use of small doses of medications like iv lidocaine, ketamine, opioids and nonsteroidal anti-inflammatory drugs (NSAID) before propofol injection and diluting the propofol solution have been tried and used to prevent injection pain $[4,5]$. Preemptive analgesia is an analgesia method with the aim of postoperative pain control with analgesic administered before pain forms due to the surgical stimuli and which reduces the severity of pain and the requirements for analgesic agents [6]. Preemptive analgesia administration ensures low postoperative pain scores among patients and reduces the amount of opioids used in the postoperative period [7,8]. NSAIDS, paracetemol and local anesthetics are commonly used to ensure preemptive analgesia $[7,9,10]$. Ibuprofen has very good analgesic and antipyretic properties and is a phenylpropionic acid derived NSAID. Due to its analgesic and anti-inflammatory properties, it is commonly used for acute and chronic inflammation and postoperative pain treatment. Oral, topical and intravenous forms are available [11]. In this research, the aim was to observe whether $400 \mathrm{mg}$ iv ibuprofen administered with the aim of preemptive analgesia had a positive effect on preventing injection pain linked to propofol administered during anesthesia induction.

\section{Material and Methods}

This study was completed with 120 patients aged from 18-65 years in ASA I-II risk groups with elective operations planned after receiving local ethics committee approval (Ordu University Ethics Committee; 2017/47) and patient consent forms. Cases who were pregnant, could not be communicated with, had psychiatric or neurologic disease, had liver, heart or renal failure, had history 
of thrombophlebitis, had allergy to NSAID medications and had history of gastrointestinal system hemorrhage were excluded from the study. Patients were randomly allocated to one of the three groups including 40 cases each using a computergenerated sequence of numbers and a sealed envelope technique: Group C (control group), Group L (lidocaine group) and Group I (ibuprofen group). The study was completed in a double blind manner by ensuring the patient and the researchers evaluating the injection pain were not aware of the medication used. All patients were administered $50 \mathrm{mg}$ ranitidine for premedication 30 minutes before the operation. Patients were monitored with electrocardiography (ECG), non-invasive blood pressure and peripheral oxygen saturation (SPO2) and then had $0.9 \% \mathrm{NaCl}$ solution infusion begun via a 20 gauge intravenous catheter in the back of the hand. Patients in Group I had $400 \mathrm{mg}$ ibuprofen from the routine postoperative analgesia protocols administered in 100 $\mathrm{ml}$ physiologic serum as an i.v. infusion over 30 minutes. A manual tourniquet was applied to the forearm and patients in Group C were administered $4 \mathrm{ml}$ physiologic serum, while patients in Group L were administered $4 \mathrm{ml} 40 \mathrm{mg}(2 \%)$ lidocaine iv. Thirty seconds later the tourniquet was loosened and $1 / 4$ of the propofol $(2.5 \mathrm{mg} /$ $\mathrm{kg}$ ) necessary for induction was administered. Ten seconds after administering propofol, all patients had pain questioned (no pain: 0 , mild pain: 1 , moderate pain: 2 , severe pain: 3 ) and arm pull reactions (no arm pull: 0 , mild reaction: 1 , moderate reaction: 2 , severe reaction: 3) assessed. Later the remaining propofol was administered and routine anesthesia protocol $(1 \mu \mathrm{g} / \mathrm{kg}$ fentanyl and $0.6-1.2 \mathrm{mg} / \mathrm{kg}$ rocuronium) was administered and the surgical procedure began.

\section{Statistical Analysis}

Statistical analysis was performed using SPSS 17.0 for Windows software (SPSS Inc., Chicago, IL, USA). Data are presented as the number of cases and (\%) for categorical variables (ASA, severity of pain, arm pull reaction, study group, etc). Descriptive analyses are presented using mean and standard deviations for ages according to the groups and gender. The Chisquare test or Fisher's exact test, where appropriate, was used to compare categorical variables in different groups. Student $t$ test was applied to compare the ages for gender. One way ANOVA was applied to compare the ages for the groups. A p-value of less than 0.05 was considered to show statistically significant results.

\section{Results}

The study included a total of 120 patients (Figure 1). The demographic data of patients is presented in Table 1. Of patients participating in the study, 63 were female $(52.5 \%)$ and 57 were male $(47.5 \%)$. The mean age of female patients was significantly high compared to male patients $(\mathrm{p}=0.037)$. The groups were similar in terms of age, gender and ASA classification. According to pain scores, the distribution of patients in the groups is shown in Table 2. In Group C there was no patient with a pain score of 0 , while this rate was 70\% in Group L and 20\% in Group I ( $p<0.05)$. In Group I, $52.5 \%$ of patients were observed to have mild pain, and this rate was significantly high compared to Group C and Group L $(\mathrm{p}<0.001)$. In Group $\mathrm{C}$ the number of patients feeling moderate or severe levels of pain was observed to be significantly high compared to Group L and Group I $(\mathrm{p}<0.001, \mathrm{p}<0.05)$. In terms of arm pull reactions, the distribution of patients is shown in Table 3. No reaction was observed in $85 \%$ of patients in Group $\mathrm{L}$ and $70 \%$ of patients in Group I (reaction scale 0 ), and these were significantly high compared to Group C $(17.5 \%)(\mathrm{p}<0.001)$. In Group C, 35\% of patients had a mild reaction, and this was significantly high compared to Group L and Group I ( $\mathrm{p}<0.001)$. The patients in Group I with moderate levels of arm pull reaction were lower compared to patients in Group $C(p=0.06)$; though this situation was not statistically significantly different, it is interpreted as being clinically significant. There was no severe arm pull reaction in any patient in Group I, though it was observed in 1 patient in Group L and 6 patients in Group C ( $\mathrm{p}<0.05)$.

Table 1: Patients demographic characteristics.

\begin{tabular}{|c|c|c|c|c|}
\hline & Group C & Group L & Group I & \multirow{2}{*}{ Total } \\
\cline { 2 - 4 } & Saline & Lidocaine & Ibuprofen & $\mathbf{n}=\mathbf{1 2 0}$ \\
\cline { 2 - 5 } & $\mathbf{n = 4 0}$ & $\mathbf{n}=\mathbf{4 0}$ & $\mathbf{n}=\mathbf{4 0}$ & $44.60 \pm 13.03$ \\
\hline Age (yr) & $45.92 \pm 13.42$ & $42.00 \pm 12.17$ & $20 / 20$ & $57 / 63$ \\
\hline Gender (M / F) & $15 / 25$ & $22 / 18$ & $23 / 17$ & $57 / 63$ \\
\hline ASA I / II & 29 -Nov & $23 / 17$ & \\
\hline
\end{tabular}

F/M: Female / Male, ASA: American Society of Anesthesiologist.

Table 2: Incidence and severity of pain.

\begin{tabular}{|c|c|c|c|}
\hline \multirow{2}{*}{ Pain Scale } & Group C & Group L & Group I \\
\cline { 2 - 4 } & Saline & Lidocaine & Ibuprofen \\
\cline { 2 - 4 } & $\mathbf{n}=\mathbf{4 0}$ & $\mathbf{n}=\mathbf{4 0}$ & $\mathbf{n = 4 0}$ \\
\hline 0 (no pain) & $0(\% 0)$ & $28(\% 70)^{\mathrm{a}, \mathrm{b}}$ & $8(\% 20)^{\mathrm{a}}$ \\
\hline 1 (mild pain) & $5(\% 12.5)$ & $7(\% 17.5)$ & $21(\% 52.5)^{\mathrm{c}}$ \\
\hline 2 (moderate pain) & $22(\% 55)^{\mathrm{d}}$ & $4(\% 10)$ & $7(\% 17.5)$ \\
\hline 3 (severe pain) & $13(\% 32.5)^{\mathrm{e}}$ & $1(\% 2.5)$ & $4(\% 10)$ \\
\hline
\end{tabular}


a: According to Group C $p<0.05$,

b: According to Group I $p<0.001$,

c: According to Grup $C$ and Group $L p<0.001$,

d: According to Grup L and Group I $p<0.001$,

e: According to Group I and Group L $p<0.05, p<0.001$

Table 3: Incidence and severity of arm pull reaction.

\begin{tabular}{|c|c|c|c|}
\hline \multirow{2}{*}{ Reaction Scale } & Group C & Group L & Group I \\
\cline { 2 - 4 } & Saline & Lidocaine & Ibuprofen \\
\cline { 2 - 4 } & $\mathbf{n = 4 0}$ & $\mathbf{n = 4 0}=\mathbf{4 0}$ \\
\hline 0 (no reaction) & $7(\% 17.5)$ & $34(\% 85)^{\mathrm{a}}$ & $28(\% 70)^{\mathrm{a}}$ \\
\hline 1 (mild reaction) & $14(\% 35)^{\mathrm{b}}$ & $5(\% 12.5)$ & $6(\% 15)$ \\
\hline 2 (moderate reaction) & $13(\% 32.5)$ & $0(\% 0)^{\mathrm{c}}$ & $6(\% 15)^{\mathrm{d}}$ \\
\hline 3 (severe reaction) & $6(\% 15)^{\mathrm{e}}$ & $1(\% 2.5)$ & $0(\% 0)$ \\
\hline
\end{tabular}

a: According to Group C $p<0.001$,

b: According to Group L and Group I $p<0.05$,

c: According to Group $C$ and Group I $p<0.05$,

d: According to Group C p:0.06, e: According to Group L and Group I p $<0.05$

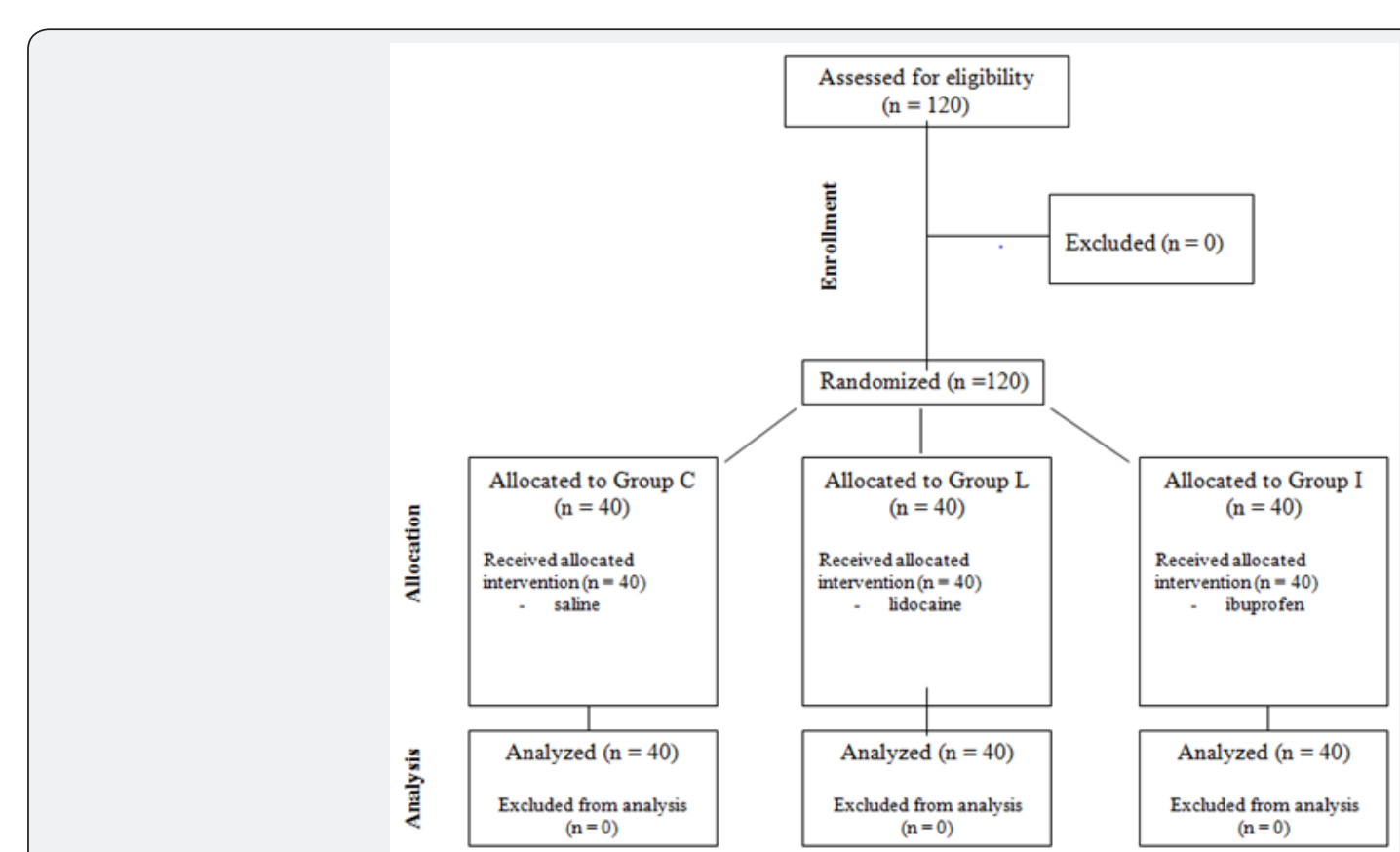

Figure 1: CONSORT flow diagram of trial.

\section{Discussion}

Though propofol injection pain is not known to be a lifethreatening complication of anesthesia, it is defined as an unpleasant situation for patients related to anesthesia [3]. According to research, the incidence risk of propofol injection pain varies from $28-90 \%$ for adults [2]. Propofol injection pain is a research area that has attracted the interest of many researchers and when the literature is examined, in 2011, 177 clinical studies including over 25,000 cases appear to be related to propofol injection pain [3]. Methods and medications applied to prevent propofol injection pain have been investigated from aspects such as causing increasing labor in the intense operating room process and financial resources. However, currently research into the topic shows propofol injection pain as a complication of anesthesia, albeit one with low morbidity, and research into the topic continues to be on the agenda $[3,12,13]$. Propofol irritates skin and mucous membranes as all phenols do. Especially bolus doses in aqueous forms cause irritation of venous adventisia during injection and cause pain [13]. Though the true mechanism of pain linked to propofol injection has not been fully explained, it is reported this pain may occur due to activation of the kallikreinkinin cascade. Activation of the kallikrein-kinin cascade and bradykinin increases contact with free nerve endings and as a result causes the formation of severe pain induced by propofol [14]. There is research available in the literature showing kallikrein inhibitors inhibit propofol injection pain $[15,16]$. 
Methods like the use of broad-diameter veins and preparation of diluted forms of propofol and administration of medications like lidocaine, opioids, magnesium and dexmedetomidine before propofol injection are methods currently used to avoid propofol injection pain $[15,17]$. As opioids and lidocaine, especially, are commonly used in routine general anesthesia induction, they are accepted as reasonable options to be administered with the aim of preventing propofol injection pain. Among all these methods, the most effective method is lidocaine administration $[18,19]$. Lidocaine may be administered before propofol or by mixing with it [1].

Picard et al. [20] in research screening randomized controlled studies identified that the most effective analgesic method was iv lidocaine administered with Bier's block before propofol injection. A study by Pang et al. identified that the use of lidocaine to avoid propofol injection pain caused $11 \%$ incidence of propofol injection pain [21]. Lidocaine is reported to stabilize the kinin cascade [1]. Additionally, it is not clearly known whether pain is prevented by the local anesthetic effect of intravenous injection of lidocaine or by a systemic effect on the central nervous system. Research on this topic by Xing et al. administered different doses of lidocaine and reported $40 \mathrm{mg}$ of lidocaine was effective in preventing propofol injection pain, with $1.5 \mathrm{mg} / \mathrm{kg}$ doses of lidocaine administration preventing pain due to both local anesthetic effect and central analgesic effect [22]. In our study in the group with iv ibuprofen administered for preemptive analgesia, the pain score 0 was higher compared to the control group, but lower compared to the lidocaine group. When assessed in terms of patients who felt severe pain, the pain score of 3 was identified to be lower in the iv ibuprofen group compared to the control group and higher compared to the lidocaine group. It appears the most effective method to prevent severe pain is lidocaine. When assessed in terms of the arm pull reaction, the highest arm pull reaction of 0 was $85 \%$ in the lidocaine group, while in $70 \%$ of Group I there was no arm pull reaction observed. When assessed in terms of severe arm pull reaction, no patient in Group I was observed to have severe arm pull reaction. Similarly in previous studies, the incidence of propofol injection pain was reported to be lower in the lidocaine pretreatment groups compared to only saline. When examined in terms of lidocaine, it is possible to say our study results are in accordance with the literature [18,19]. Another method to prevent propofol injection pain is pretreatment with ondansetron.

Pei et al. [23] in a review of PubMed, Cochrane Library and China National Knowledge Infrastructure (CNKI) researched the efficacy of ondansetron and reported that ondansetron had similar efficacy to lidocaine in reducing propofol injection pain. In recent years, there are studies in the literature on whether methods like acupuncture, heating the intravenous entry point and pretreatment with ketamine are effective in preventing propofol injection pain $[12,24]$. Some agents used to prevent propofol injection pain have the possibility of causing adverse effects related to the procedure applied to the patient in special circumstances. For example, some medications may cause shortening of the seizure duration if used during anesthesia administration for electroconvulsive therapy (ECT). As a result, it may not be possible to use the same agents for all surgical types or interventional procedures. It appears logical to note the benefit-harm ratio for the surgical procedure or intervention of agents to be used in these specific groups. Research on this topic has reported that pretreatment with dexmedetomidine reduced propofol injection pain while not affecting seizure duration [13]. There are studies in the literature about the use of paracetemol to prevent propofol injection pain.

Borazan et al. [25] reported the efficacy of paracetemol used to prevent propofol injection pain may be related to inhibition occurring in prostaglandin synthesis. The researchers compared the efficacy of iv paracetemol at $0.5 \mathrm{mg} / \mathrm{kg}, 1 \mathrm{mg} / \mathrm{kg}$ and $2 \mathrm{mg} / \mathrm{kg}$ doses with $0.5 \mathrm{mg} / \mathrm{kg}$ lidocaine and identified that the $2 \mathrm{mg} / \mathrm{kg}$ dose of paracetemol was the most effective method to prevent pain proportional to lidocaine [25]. Canbay et al. [18] reported that $50 \mathrm{mg}$ iv paracetemol administration was effective in reducing propofol injection pain; however different studies were required to determine the optimum doses. Propofol injection pain is thought to be caused by kininogens released from vein walls as a result of triggering of the local kinin cascade. For this reason, NSAIDs inhibit the prostaglandin synthesis routes in veins and are proposed to reduce propofol injection pain [1]. Many researchers have researched the efficacy of NSAIDs like lornoxicam, ketorolac and flurbiprofen to prevent propofol injection pain [26-28]. NSAIDs are reversible cyclo-oxygenase inhibitors ensuring symptomatic easing in acute and chronic inflammation. When examined in terms of NSAIDs, these studies appear to have obtained different results. Some research has found NSAIDs have high efficacy in preventing pain, while some studies have revealed low efficacy and this situation has been reported to be due to the irritant effects caused by NSAIDs during iv administration [2729]. To date it appears some other NSAIDs have been researched for reducing propofol injection pain; however there is no research in the literature on ibuprofen related to propofol injection pain.

Ibuprofen has similar analgesic effect to paracetemol and is a phenylpropionic acid derivative NSAID. Oral, topical and intravenous forms are available. Ibuprofen first entered use as a new type of NSAID in 1969 in the United Kingdom and therapeutic efficacy was easily accepted $[30,31]$. According to current product information for ibuprofen, administration duration is minimum $30 \mathrm{~min}$. More rapid infusions cause pain related to the infusion [11]. There is no research encountered in the literature on intravenous ibuprofen related to propofol injection pain. The basic foundation of our research was to observe whether iv ibuprofen administered preemptively for postoperative analgesia was effective in preventing propofol injection pain and our research results show that intravenous ibuprofen was effective in reducing propofol injection pain. However, though preemptively administered iv ibuprofen was found to be effective in preventing propofol injection pain, administration of ibuprofen diluted with 
$100 \mathrm{ml}$ fluid over $30 \mathrm{~min}$ infusion, and the possibility of infusion pain when administered more rapidly or in more concentrated form, is a limiting factor for the use of ibuprofen to reduce propofol injection pain.

\section{Conclusion}

In conclusion, we believe that knowing the effect of ibuprofen in reducing propofol injection pain for patients administered the preemptive analgesic of ibuprofen will reduce the necessity for the use of other additional medications or methods.

\section{Conflict of Interest}

The authors declare; no conflict of interest.

\section{Acknowledgement}

We special thanks to Prof. Soner ÇANKAYA from Ordu University School of Medicine Department of Biostatistics and Medical Informatics, for helping our statistical analysis.

\section{References}

1. Madan HK, Singh R, Sodhi GS (2016) Comparsion of intravenous lignocaine, tramadol and keterolac for attenuation of propofol injection pain. J Clin Diagn Res 10(7): 5-8.

2. Agarwal A, Ansari MF, Gupta D, Pandey R, Raza M, et al. (2004) Pretreatment with thiopental for prevention of pain associated with propofol injection. Anesth Analg 98(3): 683-686.

3. Wang W, Wu L, Zhang C, Sun L (2017) Is propofol injection pain really important to patients? BMC Anesthesiol 17(1): 24

4. Abdusoglu MN, Ozkocak I, Yurtlu BS, Hanci V, Okyay RD, et al. (2011) A subhypnotic dose of ketamine reduces pain associated with injection of propofol and rocuronium. Eur J Anaesthesiol 28: 229-230.

5. Kizilcik N, Menda F, Bilgen S, Keskin O, Koner O (2015) Effects of a fentanyl-propofol mixture on propofol injection pain: a randomized clinical trial. Korean J Anesthesiol 68(6): 556-560.

6. Woolf CJ, Chong MS (1993) Preemptive analgesia treating postoperative pain by preventing the establishment of central sensitization. AnestAnalg 77(2): 362-379.

7. Ahiskalioglu EO, Ahiskalioglu A, Aydin P, Yayik AM, Temiz A (2017) Effects of single-dose preemptive intravenous ibuprofen on postoperative opioid consumption and acute pain after laparoscopic cholecystectomy. Medicine (Baltimore) 96(8): e6200.

8. Pınar HU, Karaca Ö, Karakoç F, Doğan R (2017) Effects of addition of preoperative intravenous ibuprofen to pregabalin on postoperative pain in posterior lumbar interbody fusion surgery. Pain Res Manag 2017: 1030491.

9. Katz J (2001) Pre-emptive analgesia: importance of timing. Can J Anaesth 48(2): 105-114.

10. Kadıglu AG, Turker G, Gurbet A, Demirci A, Hulagu I (2013) Analgesic efficacy of pre-emptive dexketoprofen on postoperative pain after elective laparotomy. Turk J Anaesth Reanim 41(3): 75-79.

11. Bookstaver PB, Miller AD, Rudisill NC, Norris LB (2010) Intravenous ibuprofen: the first injectable product for the treatment of pain and fever. Journal of Pain Research 3: 67-79.

12. Huang L, Pan Y, Chen S, Zhang M, Zhuang X, et al. (2017) Prevention of propofol injection-related pain using pretreatment transcutaneous electrical acupoint stimulation. Turk J Med Sci 47(4): 1267-276.
13. Li X, Chen CJ, Tan F, Pan JR, Xing JB, et al. (2017) Effect of dexmedetomidine for attenuation of propofol injection pain in electroconvulsive therapy: a randomized controlled study. J Anesth 32(1): 70-76.

14. Nakane M, Iwama H (1999) A potential mechanism of propofolinduced pain on injection based on studies using nafamostat mesilate. Br J Anaesth 83(3): 397-304.

15. Tan CH, Onsiong MK (1998) Pain on injection of propofol. Anaesthesia 53: 468-476.

16. Iwama H, Nakane M, Ohmori S, Kaneko T, Kato M, et al. (1998) Nafamostat mesilate, a kallikrein inhibitor, prevents pain on injection with propofol. Br J Anaesth 81(6): 963-964.

17. Memis D, Turan A, Karamanlioglu B, Süt N, Pamukcu Z (2002) The use of magnesium sulfate to prevent pain on injection of propofol. Anesth Analg 95(3): 606-608.

18. Canbay O, Celebi N, Arun O, Karagöz AH, Sarıcaoğlu F, et al. (2008) Efficacy of intravenous acetaminophen and lidocaine on propofol injection pain. Br J Anaesth 100(1): 95-98.

19. Nathanson MH, Gajraj NM (1996) Reducing the pain on injection of propofol. Anesth Analg 82(6): 1307-1308.

20. Picard P, Tramèr MR (2000) Prevention of Pain on Injection with Propofol: A Quantitative Systematic Review. Anesth Analg 90(4): 963969.

21. Pang WW, Mok MS, Huang S, Hwang MH (1998) The analgesic effect of fentanyl, morphine, meperidine, and lidocaine in the peripheral veins: a comparative study. Anesth Analg 86(2): 382-386.

22. Xing J, Liang L, Zhou S, Luo C, Cai J, et al. (2017) Intravenous Lidocaine Alleviates the Pain of Propofol Injection by Local Anesthetic and Central Analgesic Effects. Pain Med 19(3): 598-607.

23. Pei S, Zhou C, Zhu Y, Huang B (2017) Efficacy of ondansetron for the prevention of propofol injection pain: a meta-analysis. J Pain Res 10: 445-450.

24. Jeong M, Yoon H (2016) Comparison of the effects of lidocaine preadministration and local warming of the intravenous access site on propofol injection pain: Randomized, double-blind controlled trial. Int J Nurs Stud 61: 209-218.

25. Borazan H, Erdem TB, Kececioglu M, Otelcioğlu S (2010) Prevention of pain on injection of propofol: a comparison of lidocaine with different doses of paracetamol. Eur J Anaesthesiol 27(3): 253-257.

26. Başak N, Aksoy Y, Kaydu A, Şahin ÖF (2017) Lornoxicam use to reduce the pain associated with propofol injection. Libyan J Med 12(1): 1313093.

27. Yull DN, Barkshire KF, Dexter T (2000) Pretreatment with ketorolac and venous occlusion to reduce pain on injection of propofol. Anaesthesia 55(3): 284-287.

28. Karasawa F, Ehata T, Okuda T, Satoh T (2000) Propofol injection pain is not alleviated by pretreatment with flurbiprofen axetil, a prodrug of a nonsteroidal antiinflammatory drug. J Anesth 14(3): 135-137.

29. Smith AJ, Power I (1996) The effect of pretreatment with ketorolac on pain during intravenous injection of propofol. Anaesthesia 51(9): 883885.

30. James M Ritter, Lionel D Lewis, Timothy GK Mant, Albert Ferro (2008) A Textbook of Clinical Pharmacology and Therapeutics. ( $5^{\text {th }}$ edn), Analgesics and the control of pain pp. 158.

31. Busson M (1986) Update on ibuprofen: review article. Int Med Res 14(2): 53-52. 
Your next submission with Juniper Publishers will reach you the below assets

- Quality Editorial service

- Swift Peer Review

- Reprints availability

- E-prints Service

- Manuscript Podcast for convenient understanding

- Global attainment for your research

- Manuscript accessibility in different formats

( Pdf, E-pub, Full Text, Audio)

- Unceasing customer service

Track the below URL for one-step submission https://juniperpublishers.com/online-submission.php 